\title{
Matrix Metalloproteinases Contribute to Neuronal Dysfunction in Animal Models of Drug Dependence, Alzheimer's Disease, and Epilepsy
}

\author{
Hiroyuki Mizoguchi, ${ }^{1}$ Kiyofumi Yamada, ${ }^{2}$ and Toshitaka Nabeshima ${ }^{3,4}$ \\ ${ }^{1}$ Futuristic Environmental Simulation Center, Research Institute of Environmental Medicine, Nagoya University, \\ Nagoya 464-8601, Japan \\ ${ }^{2}$ Department of Neuropsychopharmacology and Hospital Pharmacy, Nagoya University Graduate School of Medicine, \\ Nagoya 466-8560, Japan \\ ${ }^{3}$ Department of Chemical Pharmacology, Meijo University Graduate School of Pharmaceutical Sciences, Nagoya 468-8503, Japan \\ ${ }^{4}$ Comparative Cognitive Science Institutes, Meijo University, Nagoya 468-8503, Japan
}

Correspondence should be addressed to Hiroyuki Mizoguchi, h-mizo@riem.nagoya-u.ac.jp

Received 25 August 2011; Accepted 17 November 2011

Academic Editor: Fengyu Song

Copyright (C) 2011 Hiroyuki Mizoguchi et al. This is an open access article distributed under the Creative Commons Attribution License, which permits unrestricted use, distribution, and reproduction in any medium, provided the original work is properly cited.

\begin{abstract}
Matrix metalloproteinases (MMPs) and tissue inhibitors of metalloproteinases (TIMPs) remodel the pericellular environment by regulating the cleavage of extracellular matrix proteins, cell surface components, neurotransmitter receptors, and growth factors that mediate cell adhesion, synaptogenesis, synaptic plasticity, and long-term potentiation. Interestingly, increased MMP activity and dysregulation of the balance between MMPs and TIMPs have also been implicated in various pathologic conditions. In this paper, we discuss various animal models that suggest that the activation of the gelatinases MMP-2 and MMP-9 is involved in pathogenesis of drug dependence, Alzheimer's disease, and epilepsy.
\end{abstract}

\section{Introduction}

Matrix metalloproteinases (MMPs) function to remodel the pericellular environment, primarily by cleaving extracellular matrix (ECM) proteins [1]. This family of enzymes contains more than 20 members, all of which require $\mathrm{Zn}^{2+}$ for their enzymatic activities. Several MMPs, including all of the membrane-anchored versions, contain a furin motif (MMP11, MMP-14, MMP-15, and MMP-16, among others) that mediates intracellular activation of the proteinase prior to its appearance outside of the cell. The activation of the other MMPs requires extracellular proteolytic processing of the secreted zymogens into the active enzymes, a process performed by MMPs or specific proteinases [2, 3].

MMP activity is regulated by interactions with tissue inhibitors of metalloproteinases (TIMPs), a family of multifunctional secreted proteins (TIMP-1-4) that promote growth and regulate the cell cycle in various cell types $[1,4]$. Regional and cell-specific expression of MMPs and TIMPs has been detected at various stages of cerebellar development, which may be related to granular cell migration, arborization of Purkinje cells, and synaptogenesis [5]. The regulation of MMPs expression and activity appears to be a very complex process [6]. The expression is regulated at the level of transcription by a variety of growth factors, cytokines, and chemokines although posttranscriptional and epigenetic modification may also contribute.

In an exemplary case of MMP-9, there are a number of gene regulatory sequences driving the MMP-9 expression, with AP1 and NF- $\kappa$ B being the most prominent. MMP-9 is produced by cells in an inactive form. Fully active protein is produced by disruption of the cysteine-zinc interaction and enzymatic removal of the propeptide. The activation of proenzyme is controlled by a cascade of steps involving other MMP and the plasmin system. Pro-MMP-9 can form a complex with TIMP-1, which involves interaction of the C-terminal (noninhibitory) domain of pro-MMP-9 and the C-terminal (noninhibitory) domain of TIMP-1. Moreover, 
low-density receptor-related protein can act as a receptor for MMP-9, which mediates internalization and degradation of the enzyme.

Of note, extensive cellular migration and remodeling of the ECM are necessary for neural development [7]. It was recently demonstrated that neuronal TIMP-1 $[8,9]$ and MMP-9 [10] are regulated by synaptic activity, suggesting that the balance between MMPs and TIMPs contributes to activity-dependent reorganization of the neuronal architecture and synaptic physiology. Additionally, MMPs and TIMPs modulate pathophysiologic functional and structural remodeling of the cellular architecture, primarily by regulating the cleavage of ECM proteins, the bioavailability of growth factors and cytokines, and shedding of membrane receptors $[1,3]$. Indeed, MMPs have been linked to various pathologic conditions of the central nervous system (CNS), including ischemia, Alzheimer's disease, multiple sclerosis, Parkinson's disease, and malignant glioma. In particular, altered regulation of MMP-2 and MMP-9 has been linked to several nervous system disorders [1]. Therefore, studies of MMPs as key enzymes in both normal and abnormal brain functioning represent a rapidly emerging field.

Keeping these background in mind, we have been investigating the physiologic and pathophysiologic roles for MMP/TIMP in learning and memory, drug dependence, Alzheimer's disease, and epilepsy. In this paper, we discuss potential roles of MMPs-especially MMP-2 and MMP-9-in physiologic and pathophysiologic neural processes.

\section{MMPs in Emotion and Memory}

MMP-9 expression is upregulated, and the protein becomes proteolytically active during the maintenance phase of longterm potentiation (LTP) at CA3-CA1 synapses in the hippocampus [11]. Conversely, TIMP-1 abolishes MMP-9-dependent long-lasting LTP in the prefrontal cortex [12]. These reports suggest a role for MMP-9/TIMP-1 system in neuronal plasticity such as LTP and memory.

FN-439, a broad-spectrum metalloproteinase inhibitor, disrupts reconsolidation of fear memories, which is related to emotionality in a reactivation-dependent manner [13]. Infusing an MMP inhibitor into the dorsal hippocampus disrupted acquisition of spatial memory during the Morris's water maze test [14]. Hippocampal MMP-9 expression transiently increased during memory acquisition in water maze tests, and inhibition of MMP activity with MMP-9 antisense oligonucleotides and an MMP inhibitor altered long-term potentiation and prevented the acquisition of spatial memories during the Morris's water maze test [15]. Conversely, TIMP-1-deficient mice fail to acquire an odor-conditioned learning task, suggesting that the disruption of MMP/TIMP balance disrupt physiologic function such as learning [16]. Interestingly, tissue plasminogen activator and MMPs are also associated with anxiety-like behavior and memory formation, with some studies demonstrating that tissue plasminogen activator in the amygdala promoted stress-induced synaptic plasticity and anxiety-like behavior $[17,18]$. Thus, expression of these proteases may be critical for emotion and
TABLE 1: Summary of behavioral changes related to emotional responses in MMP- $2^{(-/-)}$and MMP- $9^{(-/-)}$mice.

\begin{tabular}{lccc}
\hline Test & Parameters & $\begin{array}{c}\text { MMP- } \\
9^{(-)-}\end{array}$ & $\begin{array}{c}\text { MMP- } \\
2^{(-)-}\end{array}$ \\
\hline & Outer sector crossing & \pm & \pm \\
& Inner sector crossing & \pm & \pm \\
Open-field test & Time in inner sector & \pm & \pm \\
& Number of rearing & $\Uparrow$ & \pm \\
& Number of grooming & \pm & \pm \\
& Start latency & $\Downarrow$ & $\Downarrow$ \\
\hline \multirow{4}{*}{ Elevated plus-arm } & Time in open arm & $\Uparrow$ & \pm \\
maze test & Open arm entries & $\Uparrow$ & \pm \\
& Time in closed arm & $\Downarrow$ & \pm \\
& Closed arm entries & \pm & \pm \\
\hline
\end{tabular}

Behavioral changes in MMP-9 $9^{(-/-)}$mice indicate that endogenous MMP-9 play a role in emotional behaviors.

\pm : no change; $\Uparrow$ : significant increase versus wild-type mice; $\Downarrow$ : significant decrease versus wild-type mice. Modified from Mizoguchi et al. [21].

memory, and deletion of MMPs in mice may induce abnormalities in behavioral tests.

On the other hand, activity-dependent changes in the ECM are thought to alter synaptic architecture and physiology such that the efficiency of synaptic transmission is affected. A recent study identified MMP-9 as a physiologic regulator of N-methyl-D-aspartate (NMDA) receptor-dependent synaptic plasticity and memory. These effects of MMP-9 on NMDA receptors are not mediated by changes in the overall ECM structure or direct cleavage of NMDA receptor subunits, but rather involve an integrin $\beta 1$-dependent pathway [19]. NMDA receptors are important mediators of synaptic plasticity that are central to the neurobiological underpinnings of emotionality, learning, and memory [20]. Thus, MMPs may play critical roles in emotion and memory by extracellularly regulating NMDA receptor signaling.

\subsection{Emotional and Cognitive Behavioral Phenotypes in MMP-} $2^{(-/-)}$and MMP-9 $9^{(-/-)}$Mice. We investigated the roles of MMP-2 and MMP-9 in emotional and cognitive function using mice with targeted deletions of the $M M P-2$ or $M M P$ 9 genes [21]. Emotional changes in MMP-2 ${ }^{(-/-)}$manifested as more frequent rearing in the open-field test and increased time in the open arms and more open arm entries in the elevated plus-arm maze test. Unlike MMP- $2^{(-/-)}$mice, when MMP- $9^{(-/-)}$mice were exposed to mildly stressful situations, they showed behavioral responses that indicated reduced anxiety (Table 1). Thus, endogenous MMP-9 may contribute to signaling pathways that modulate emotional behaviors.

MMP-9 $9^{(-/-)}$mice also showed impaired long-term object recognition and fear memory, whereas spontaneous alternation behavior in the Y-maze test and short-term memory were normal [21]. MMP-2 $2^{(-/-)}$mice do not have any learning or memory phenotypes. These results suggest that MMP9 plays a role in the formation of long-term memories but not short-term memories (Table 2). Furthermore, Nagy et al. [11] showed that MMP-9 null-mutant mice displayed 
TABLE 2: Summary of impaired learning and memory parameters induced by MMP inhibition.

\begin{tabular}{|c|c|c|c|}
\hline Test (type of memory) & MMP inhibitor & MMP-9 $9^{(-/-)}$ & MMP-2(-/-) \\
\hline Y-maze test (short-term memory) & N.D. & \pm & $\Downarrow$ \\
\hline Novel-object recognition test (short-term object memory) & N.D. & \pm & \pm \\
\hline Novel-object recognition test (long-term object memory) & N.D. & $\Downarrow$ & \pm \\
\hline Conditioned fear memory test (long-term fear memory) & $\Downarrow$ & $\Downarrow$ & \pm \\
\hline Morris's water maze test (hippocampus-dependent spatial memory) & $\Downarrow$ & N.D. & N.D. \\
\hline
\end{tabular}

Performance of MMP-9(-/-) mice in various memory test indicates impairments of long-term memory in the mutant mice.

\pm : no change; $\Downarrow$ : significant decrease versus control or wild-type mice; N.D.: not determined.

Modified from [13-15, 21].

TABLE 3: Changes in MMP and TIMP expression levels and activities induced by drugs of abuse.

\begin{tabular}{|c|c|c|c|c|c|c|}
\hline Drug & MMP-2 & MMP-9 & TIMP-2 & MMP-3 & Method (expression/activity) & References \\
\hline Methamphetamine (single treatment) & \pm & \pm & \pm & N.D. & mRNA/activity & {$[32,33]$} \\
\hline Methamphetamine (repeated treatment) & $\Uparrow$ & $\Uparrow$ & $\Uparrow$ & N.D. & mRNA/protein/activity & {$[32,33]$} \\
\hline Cocaine & N.D. & $\Uparrow$ & N.D. & \pm & Activity & {$[38]$} \\
\hline Morphine & $\Uparrow$ & N.D. & $\Uparrow$ & N.D. & Protein/activity & Our unpublished data \\
\hline \multirow{2}{*}{ Alcohol } & N.D. & $\Uparrow$ & N.D. & N.D. & Protein & {$[39]$} \\
\hline & $\Uparrow$ & $\Uparrow$ & $\Downarrow$ & N.D. & Protein/activity & {$[40]$} \\
\hline
\end{tabular}

$\pm:$ no change; $\Uparrow$ : significant increase versus control mice; N.D.: not determined.

a significant deficit in long-term hippocampus-dependent memories for context conditioning but not cued conditioning. These results indicate that MMPs play critical roles in synaptic plasticity and hippocampal-dependent memory and that inhibiting MMP activity may compromise the ability of the dorsal hippocampus to reconfigure ECM molecules and interfere with spatial memory acquisition. Although no major defects in brain structures were observed with histochemical examinations, it is possible that neuroadaptation and compensatory mechanisms activated in response to tarsgeted deletions of $M M P-2$ and $M M P-9$ contributed to the emotional and cognitive phenotypes in the mutant mice.

\section{MMPs in Drug Dependence}

Drug dependence is a complex phenomenon that, in some cases, is mediated by neural plasticity and remodeling of specific brain circuits after repeated exposure to a drug [22, 23]. For example, methamphetamine (METH), a commonly abused drug, causes both acute and long-lasting effects on psychomotor behavior [24-26]; these effects are associated with increased extracellular dopamine levels in the brain, owing to facilitated dopamine release from presynaptic nerve terminals and inhibited dopamine reuptake [27]. The cellular and molecular mechanisms underlying drug dependence are thought to include processes similar to those operating in other forms of synaptic plasticity, such as learning and memory $[22,23,28]$. Nevertheless, although repeated treatment with cocaine or amphetamines alters neural morphology and synaptic connectivity in the mesolimbic system $[29,30]$, the mechanisms underlying psychostimulant-induced remodeling of synaptic structures have not been determined [31].
3.1. METH-Induced Changes in MMP and TIMP Expression in the Brain. We demonstrated that repeated administration of METH led to behavioral sensitization, which was accompanied by the induction of MMP-2, MMP-9, and TIMP2 expression in various regions of the brain, including the frontal cortex and nucleus accumbens (NAc; Table 3) [3234]. Our data also showed that MMP-2 and MMP-9 were expressed in neurons and glial cells. Repeated, but not single, METH doses also induced TIMP-2 mRNA and protein expression in neurons [32]. Previous studies also suggested that the MMP/TIMP system is active in neuronal and glial cells and that the expression levels and cellular localization of these proteins are regulated according to developmental and/or functional status of the brain $[11,35]$. For instance, during axonal extension, MMPs are located at growth cone tips, where they mediate attachment and detachment of neurons to the matrix substratum, whereas MMPs mediate process extension in oligodendrocytes, suggesting that the regulation of MMPs is an important component of synaptogenesis [2]. Accordingly, MMP-2 and MMP-9 may contribute to structural and functional alterations in the brain following repeated exposure to METH.

3.2. Roles of the MMP/TIMP System in METH-Induced Behavioral Sensitization and Reward. We demonstrated that METH-induced behavioral sensitization and conditioned place preference (CPP) - a measure of the rewarding effect of drugs-were markedly attenuated in MMP- $2^{(-/-)}$and MMP-9 $9^{(-/-)}$mice compared with wild-type mice [33], and doxycycline, inhibitor of MMP-2 and MMP-9, blocked METH-induced behavioral sensitization and CPP (Table 4) [32]. Antisense TIMP-2 oligonucleotides (TIMP-AS) inhib- 
TABLE 4: Summary of changes in addictive behaviors and dopamine release in response to inhibition of the MMP/TIMP system.

\begin{tabular}{|c|c|c|c|c|c|c|}
\hline Test & Drug & TIMP-2-AS & $\begin{array}{c}\text { Doxycycline, FN-439 } \\
\text { (MMP inhibitor) }\end{array}$ & $\mathrm{MMP}-2^{(-/-)}$ & MMP-9 $9^{(-/-)}$ & References \\
\hline $\begin{array}{l}\text { Hyperlocomotion } \\
\text { (single treatment) }\end{array}$ & Methamphetamine & \pm & \pm & \pm & \pm & {$[32,33]$} \\
\hline $\begin{array}{l}\text { Locomotor sensitization } \\
\text { (repeated treatment) }\end{array}$ & Methamphetamine & $\Uparrow$ & $\Downarrow$ & $\Downarrow$ & $\Downarrow$ & {$[32,33]$} \\
\hline \multirow{2}{*}{$\begin{array}{l}\text { Conditioned place preference } \\
\text { (rewarding effect, and } \\
\text { drug-associated memory) }\end{array}$} & Methamphetamine & N.D. & $\Downarrow$ & $\Downarrow$ & $\Downarrow$ & {$[32,33]$} \\
\hline & Cocaine & N.D. & $\Downarrow$ & N.D. & N.D. & {$[37]$} \\
\hline Self-administration & Alcohol & N.D. & $\Downarrow$ & N.D. & N.D. & {$[41]$} \\
\hline Dopamine release & Methamphetamine & $\Uparrow$ & $\Downarrow$ & $\Downarrow$ & $\Downarrow$ & {$[32,33]$} \\
\hline
\end{tabular}

\pm : no change; $\Uparrow$ : significant increase versus control mice; $\Downarrow$ : significant decrease versus control mice; N.D.: not determined.

TIMP-2-AS: antisense TIMP-2 oligonucleotides.

ited METH-induced increases in TIMP-2 protein expression and enhanced locomotor sensitization, effects that were associated with potentiation of METH-induced dopamine release in the NAc (Table 4) [33]. Accordingly, a net increase in MMP activity may be responsible, at least in part, for the development of METH-induced behavioral sensitization and structural and functional changes in the brain that underlie METH dependence. Indeed, inhibiting MMPs altered functional and structural correlations of deafferentation-induced sprouting, such as remodeling in the dentate gyrus of the hippocampus [36]. Interestingly, Brown et al. [37] demonstrated that MMPs were involved in creating cocaine-associated contextual memories and that reconsolidation of these memories was disrupted by an MMP inhibitor. Further, the authors showed that an MMP inhibitor impaired the expression of memory in response to a cocaine-associated cue after extinction. In addition, elevated MMP-9 activity in the prefrontal cortex contributed to synaptic remodeling that was important for the reactivation of a cocaine-related memory or, alternatively, the modification of a competing extinction memory during reinstatement [38]. Moreover, MMP-9 is elevated in serum of alcohol abusers [39], alcohol treatment increased MMP-1, -2, and -9 activity, and decreased TIMP1 and -2 in the brain microvascular endothelial cells [40], and inhibiting MMPs attenuated ethanol self-administration [41]. These reports suggest that MMPs may also have a crucial role in drug-associated memory. Because the neurologic underpinnings of learning and memory are closely tied to the development of aberrant behaviors related to drug abuse and addiction [22, 23, 28], MMP-2 and MMP-9 expression may be critical in the acquisition of METH-induced CPP.

Recently, it was reported that MMP-9 expression does not affect METH-induced neurotoxicity and instead contributes to neural remodeling [42]. Further, Conant et al. [43] demonstrated that METH induced ICAM-5 cleavage in murine hippocampus and striatum, which was accompanied by increased MMP-9 protein levels. The authors also showed that the N-terminal ICAM-5 ectodomain stimulated phosphorylation of cofilin via an integrin $\beta 1$-dependent mechanism that was linked to spine maturation. Although additional studies are necessary, these data implicate MMPs in synaptic plasticity associated with drug dependence.

3.3. Roles of the MMP/TIMP System in Dopamine Neurotransmission. Behavioral changes induced by METH are linked to its capacity to elevate extracellular dopamine levels by redistributing dopamine from synaptic vesicles to the cytosol and promoting reverse transport $[44,45]$. METH-induced behavioral sensitization is associated with enhanced METHinduced increases in extracellular dopamine levels in the NAc $[27,45]$. Compared with wild-type mice, MMP-2 ${ }^{(-/-)}$ and MMP- $9^{(-/-)}$mice showed attenuated METH-induced dopamine release in the NAc, behavioral sensitization, and CPP [33]. Sensitization of METH-induced dopamine release in the NAc was enhanced and reduced by TIMP-AS and inhibitors of MMP-2 and MMP-9, respectively. In contrast, infusion of purified human MMP-2 into the NAc significantly potentiated METH-increased dopamine release [32]. Reduced $\left[{ }^{3} \mathrm{H}\right]$ dopamine uptake into striatal synaptosomes was observed in wild-type mice after repeated METH treatment, whereas these changes were significantly attenuated in MMP-2 ${ }^{(-/-)}$and MMP-9(-/-) mice [33]. Reverse activation and internalization of plasmalemmal dopamine transporter are involved in METH-induced increases in extracellular dopamine levels $[44,46]$. These results suggest that MMP2 and MMP-9 play crucial roles in METH-induced behavioral sensitization and reward by regulating METH-induced dopamine release and uptake via dopamine transporters in the NAc.

The sensitivity of dopamine receptors to endogenous and exogenous ligands is an important factor in physiologic and pathologic dopamine neurotransmission, and reduced signaling via dopamine D2 receptors may be an important neuroadaptation in cocaine addiction [47]. G protein signaling in the frontal cortex may play a crucial pathologic role in cocaine sensitization and drug seeking [48]. We have also shown that dopamine receptor agonist-stimulated $\left[{ }^{35} \mathrm{~S}\right] \mathrm{GTP} \gamma \mathrm{S}$ binding is reduced in the frontal cortex of rats sensitized to METH. TIMP-AS and an MMP-2/ MMP-9 inhibitor, respectively, potentiated and attenuated 
TABLE 5: MMP protein expression in the brains of patients with Alzheimer's disease.

\begin{tabular}{lcc}
\hline MMP & Cell types & References \\
\hline MMP-2 & Blood vessels and white matter glia & {$[63,64]$} \\
MMP-3 & Neuron and occasional plaque & {$[64]$} \\
MMP-9 & $\begin{array}{c}\text { Neuron, neurofibrillary tangle, } \\
\text { plaque, and vessel wall }\end{array}$ & {$[62-65]$} \\
\hline
\end{tabular}

the reduction in dopamine D2 receptor agonist-stimulated $\left[{ }^{35} \mathrm{~S}\right] \mathrm{GTP} \gamma \mathrm{S}$ binding. Repeated METH treatment also reduced dopamine D2 receptor agonist-stimulated $\left[{ }^{35} \mathrm{~S}\right] \mathrm{GTP} \gamma \mathrm{S}$ binding in wild-type mice, whereas these changes were significantly attenuated in $\mathrm{MMP}_{-} 2^{(-/)}$and MMP- $9^{(-/)}$mice [32]. These results suggest that MMPs and TIMPs are involved in METH-induced dysregulation of dopamine release and receptor signaling. Because dopamine D2 receptors function in feedback inhibition of dopamine release $[49,50]$, downregulated signaling may enhance METH-induced increases in extracellular dopamine levels.

\section{MMPs in Alzheimer's Disease}

Alzheimer's disease is characterized by the deterioration of cognitive and mental function. The pathology of Alzheimer's disease involves the formation of extracellular deposits of amyloid beta $(A \beta)$ peptide, leading to neuritic plaques and neurofibrillary tangles in the cortex and hippocampus [51, 52]. The neurotoxicity of $A \beta$ has been clearly demonstrated in vitro and in vivo and shown to involve oxidative stress, perturbed intracellular calcium homeostasis, and activation of apoptosis [53], and neural uptake of extracellular $A \beta$ [54].

Intrahippocampal injections of $A \beta$, including $A \beta 1-40$, $\mathrm{A} \beta 1-42$, and $\mathrm{A} \beta 25-35$, have been shown to induce hippocampal damage, learning and memory deficits [55-57], and impairment of the cholinergic system, which contribute to cognitive deficits associated with aging and neurodegenerative diseases [58-60]. Whereas plaques and amyloid fibrils are thought to resist proteolytic degradation, certain proteases may be involved in endogenous mechanisms that clear plaques. A recent study showed that intracerebroventricular infusion of $\mathrm{A} \beta 1-42$ induced learning deficits after 12 weeks in 9-month-old mice but not 2.5-month-old mice, suggesting that $A \beta$ infusion resulted in age-dependent and delayed learning deficits independent of $\mathrm{A} \beta$ deposition and inflammation [61].

Interestingly, MMP-9 levels are increased in the brains of patients with Alzheimer's disease (Table 5) [62-65]. MMP9 is expressed in the cytoplasm of neurons, neurofibrillary tangles, senile plaques, and vascular walls in brain tissue from patients with Alzheimer's disease [65]. Moreover, MMP9 expression is induced in astrocytes in the presence of A $\beta$ peptide [66]. Although MMP-9 cleaves the A $\beta$ peptide at several sites $[62,67]$, its role in $A \beta$-induced cognitive dysfunction and neurotoxicity is unclear.

4.1. A -Induced MMP Expression in the Brain. Previous study has revealed that both MMP-2 and MMP-9 are induced by the presence of $A \beta[67]$ and highly expressed and secreted by astrocytes $[68,69]$. Accordingly, we investigated whether MMP-2 and MMP-9 activities were induced by intracerebroventricular injections of $A \beta 25-35$ and $A \beta 1-40$ using gel zymography [70]. Intracerebroventricular injection of $A \beta 25$ $35, \mathrm{~A} \beta 1-40$, or $\mathrm{A} \beta 1-42$ transiently and dose-dependently increased hippocampal MMP-9 activity and protein expression, whereas MMP-2 was not affected. The injection of a control peptide, A $\beta 40-1$, had no effect on MMP-9 activity and protein expression in the hippocampus, suggesting that the induction of MMP-9 following intracerebroventricular $\mathrm{A} \beta$ injection is not due to nonspecific response to brain damage by the insertion of injection needle. Immunohistochemistry revealed increased expression of MMP- 9 in both neurons and glial cells in the hippocampus after $\mathrm{A} \beta$ administration [70]. When gelatinase activity was analyzed in the hippocampus following intracerebroventricular injection of A $\beta 1-40$ using in situ zymography, markedly increased activity was detected in the hippocampal molecular layer in the $A \beta 1-40$-treated group compared with the $A \beta 40-1$ injected group [70].

Moreover, we found that pretreatment with MK-801 inhibited the $A \beta$-induced increase in MMP-9 expression, indicating that activation of NMDA receptors is involved in A $\beta$-induced expression of MMP-9 in vivo [70].

4.2. MMP Inhibition and Reduced Aß-Induced Cognitive Impairment and Neurotoxicity. Because MMP-9 has been reported to cleave insoluble $A \beta$ in vitro [67], it was thought that MMP inhibitors may potentiate $A \beta$-induced cognitive dysfunction and neurotoxicity. Our findings do not support this concern, however. In fact, treatment with GM6001, an MMP inhibitor, ameliorated $\mathrm{A} \beta$-induced impairment of recognition memory, suggesting that transient increases in hippocampal MMP-9 activity are associated with the development of $\mathrm{A} \beta$-induced cognitive deficits. The findings obtained with a pharmacologic inhibitor were supported by results showing that intracerebroventricular injection of A $\beta 1-40$ impaired recognition memory in wild-type mice but not MMP-9 ${ }^{(-/-)}$mice [70]. Therefore, even if MMP9 degrades $\mathrm{A} \beta$ or amyloid plaques, it may nonselectively degrade ECM proteins and neural membranes, leading to neuronal dysfunction and cognitive impairment.

Recent evidence has linked MMPs to various pathologic conditions in the CNS, including ischemia, multiple sclerosis, Parkinson's disease, and malignant glioma. This implies that in addition to degrading ECM proteins, MMPs may regulate cell death. In fact, recent studies have indicated that MMP-9 has direct neurotoxic effects [71]. We demonstrated that MMP inhibitor II, which is reportedly highly selective for MMP-2 and MMP-9 [72], blocks $A \beta$-induced release of lactate dehydrogenase in primary cultured neurons, indicating that MMP-9 contributes to $A \beta$-induced neuronal cell death. $A \beta$-induced neurotoxicity was markedly reduced in primary cultured cortical neurons from MMP- $9^{(-/-)}$ mice compared with neurons from wild-type mice. These results suggest that MMP-9 expression in the hippocampus following $A \beta 1-40$ infusion had a causal role in the resulting neurotoxicity and cognitive impairment. On the basis of our 
TABLE 6: Summary of changes in brain MMP expression levels and activities induced by chemical stimulants.

\begin{tabular}{|c|c|c|c|c|c|c|}
\hline Drug & Dose $(\mathrm{mg} / \mathrm{kg})$ & Seizure intensity & MMP-2 & MMP-9 & Method (expression/activity) & References \\
\hline \multirow{4}{*}{ Pentylene-tetrazole } & 40 & Ear and facial twitching & \pm & \pm & Activity & {$[86]$} \\
\hline & 40 & Kindled seizure (tonic convulsion) & \pm & $\Uparrow$ & Protein/activity & {$[86]$} \\
\hline & 60 & Tonic convulsion & \pm & \pm & Activity & [86] \\
\hline & 50 & Generalized seizures & N.D. & $\Uparrow$ & mRNA & {$[79]$} \\
\hline \multirow{3}{*}{ Kainic acid } & $9-10$ & Tonic convulsion & N.D. & $\Uparrow$ & Protein/activity & {$[71,83]$} \\
\hline & 10 & Status epilepticus & N.D. & $\Uparrow$ & mRNA & {$[80]$} \\
\hline & 10 & Convulsion & $\Uparrow$ & $\Uparrow$ & Activity & {$[82]$} \\
\hline Pilocarpine & 325 & Status epilepticus & \pm & $\Uparrow$ & Protein/activity & {$[84]$} \\
\hline Bicuculline & $1.5-3.0$ & Convulsion & $\Uparrow$ & $\Uparrow$ & Activity & {$[82]$} \\
\hline
\end{tabular}

$\pm:$ no change; $\Uparrow$ : significant increase versus control mice; N.D.: not determined.

findings, it is speculated that $\mathrm{A} \beta$-induced secondary dysfunction such as MMP activation could result in learning deficits by impairing synaptic function in the hippocampus. Our findings highlight the contribution of neural/glial MMP-9 to $A \beta$-induced neurotoxicity and cognitive impairment and support the idea that for highly selective MMP-9, inhibitors may reduce deleterious proteolytic activity and neuronal death in Alzheimer's disease.

\section{MMPs in Epilepsy}

Seizures cause brain injury via a number of mechanisms that may contribute to neurologic and cognitive deficits in patients with epilepsy. Although seizures induce neuronal death in some situations, they also produce nonlethal pathophysiologic effects on neuronal structures and functions [73]. Kindling is an experimental epilepsy model in which repeated electrical or chemical stimulation of certain forebrain structures triggers progressively more intense electroencephalographic and behavioral seizure activity [7476]. Once established, kindling results in a permanent state of seizure susceptibility, which may manifest as spontaneous epileptiform seizures $[76,77]$.

Serum MMP-9 levels and the ratio of MMP-9 to TIMP-1 are elevated in children with various febrile seizures and convulsive status epilepticus [78]. Moreover, expression levels of MMP-9 mRNA increased in response to neuronal depolarization in the rat hippocampus [79], and MMP-9 mRNA was transported to dendrites and synapses in the hippocampal dentate gyrus of kainic acid-treated rats [80]. Jourquin et al. [71] used organotypic cultures to demonstrate increased release and activity of MMP-9 after stimulation with neurotoxic kainate and reduced neuronal cell death following MMP-9 inhibition. TIMP-1 mRNA is also increased in dentate gyrus following seizures, and elevated TIMP-1 mRNA and protein are measured in the hippocampus with seizure [8]. TIMPs are produced by microglia and astrocytes located in cortex and white matter and may play a role in neural regeneration depending upon the degree of expression and the time since injury [81]. Although MMP-9/TIMP-1 is expressed in response to neural activity in some models of epileptogenesis [82-85], the pathophysiologic and etiologic roles of this metalloproteinase, including potential molecular targets during kindling seizure development, are not clear.
5.1. MMPs in Kindled Seizure. Mice administered a single pentylenetetrazole (PTZ) dose $(20-40 \mathrm{mg} / \mathrm{kg})$ exhibit ear and facial twitching or, at times, convulsive twitching axially throughout the body. PTZ at a dose of $60 \mathrm{mg} / \mathrm{kg}$ induces seizures characterized by tonic convulsion, jumping, and wild running. Repeated administration of PTZ at a dose of $40 \mathrm{mg} / \mathrm{kg}$ produces chemical kindling, a phenotype associated with a progressive increase in the seizure score. Repeated PTZ treatment increased MMP-9 expression in the hippocampus for at least $24 \mathrm{~h}$ after the final dose (Table 6). On the other hand, hippocampal MMP-9 levels were not affected in mice that showed convulsive seizures in response to a single $60 \mathrm{mg} / \mathrm{kg}$ PTZ dose. No changes in hippocampal MMP-2 levels were detected following acute or repeated PTZ treatment [86].

We investigated the role of MMP-9 in PTZ-induced kindled seizure using MMP- $9^{(-/-)}$mice. No difference in the severity of tonic seizures induced by a single PTZ treatment was observed between wild-type and MMP- $9^{(-/-)}$mice. Repeated PTZ treatment induced kindled seizure in wildtype mice, whereas PTZ-treated MMP- $9^{(-/-)}$mice showed a marked delay in kindling development although progression of kindled seizure was eventually observed. These results demonstrate that deletion of MMP-9 attenuated PTZ-induced kindled seizure [86] and agreed with recent findings showing that the synaptic pool of MMP-9 is a critical determinant of seizure development [83]. We also showed that mossy fiber sprouting induced by repeated PTZ treatment was reduced in MMP- $9^{(-/-)}$mice compared with wild-type mice, suggesting that increased hippocampal MMP-9 activity is associated with the sprouting of mossy fibers [86].

5.2. MMP-9-Mediated Maturation of Brain-Derived Neurotrophic Factor and the Development of PTZ-Induced Kindling. Brain-derived neurotrophic factor (BDNF) has been shown to be a potent morphoregulator that controls axon branching and activity-dependent refinement of synapses $[87,88]$. BDNF also plays a role in epileptogenesis and kindled seizure. A recent study showed that high-frequency neuronal activity controlled the ratio of extracellular proBDNF to mature BDNF by regulating the secretion of extracellular proteases [89]. Of particular note for this paper, proforms of neurotrophin are cleaved and activated by 
MMPs [2, 3, 90], and MMP-9 converts pro-BDNF to mature BDNF, resulting in tropomyosin-related kinase B $(\operatorname{TrkB})$ activation $[2,91]$.

Repeated PTZ treatment increased BDNF mRNA and protein levels in the hippocampi of kindled wild-type mice. MMP- $9^{(-)}$mice showed reduced levels of mature BDNF during the early stage of kindling, which may explain slower kindling development in the mutant animals. During the later kindling stage, however, mature BDNF levels were similar in MMP-9 $9^{(-/)}$and wild-type mice. These results suggest that MMP-9 plays a role in kindling development by converting pro-BDNF to mature BDNF in the hippocampus, whereas other factors mediate BDNF maturation during the later kindling stage. This may explain why the seizure response curves for MMP- $9^{(-/-)}$and wild-type mice differed more at the early stage than at the later stage of kindling [86].

To prove the assumption described above, the BDNF scavenger TrkB-Fc was microinjected into the right ventricle before each PTZ treatment. The treatment significantly suppressed the development of kindling in wild-type mice, whereas no effect was observed in MMP- $9^{(-/-)}$mice. On the other hand, bilateral injections of pro-BDNF into the hippocampal dentate gyrus significantly enhanced kindling in wild-type mice but not MMP-9 $9^{(-)}$mice. These findings suggest that MMP-9 is involved in the progression of behavioral phenotypes in kindled mice owing to conversion of proBDNF to mature BDNF in the hippocampus. These results likely reflect a relationship between MMP-9 expression and mature BDNF levels in the development of PTZ-induced kindling [86].

Extracellular BDNF stimulates TrkB receptors on the hilar segment of the mossy fiber to induce axonal branching, which may create hyperexcitable dentate circuits [92]. Thus, PTZ-induced kindling may be aggravated through synaptic transformation, including mossy fiber sprouting induced by prolonged MMP-9 activation and subsequent increase in mature BDNF levels. These findings suggest the contribution of neural/glial MMP-9 to epileptogenesis via its activity on extracellular macromolecules such as BDNF.

\section{Conclusion}

As reviewed in this paper, research has begun to elucidate the roles of MMPs in physiologic processes and acute and chronic CNS disorders. Proteolytic mechanisms regulate various developmental and homeostatic processes, whereas inappropriate proteolysis causes or exacerbates a number of CNS disorders. Our previous studies suggest that MMP-9 plays a role in emotional and cognitive behaviors, which may be related to activity-dependent synaptic plasticity and brain development. Furthermore, MMP-9 expression appears to play a crucial role in METH-induced behavioral sensitization by modulating the function of such plasma membrane proteins as dopamine receptors and transporters. MMP-9 also contributes to $A \beta$-induced neuronal dysfunction and cognitive impairment by nonspecifically destroying the extracellular matrix and neural membranes. Finally, MMP-9 is involved in the progression of behavioral phenotypes in kindled mice by converting pro-BDNF to mature BDNF in the hippocampus. These results suggest that MMP overexpression is associated with structural and functional changes in the cerebral cortex and mesocorticolimbic system, leading to abnormal behaviors following drug treatment. In addition to contributions to various diseases, MMPs have many physiologic roles in such processes as neurogenesis related to memory formation and emotion. More research is needed to understand the diverse roles of these proteases and their potential as therapeutic targets.

\section{Abbreviations}

$\begin{array}{ll}\text { A } \beta: & \text { Amyloid beta } \\ \text { BDNF: } & \text { Brain-derived neurotrophic factor } \\ \text { CNS: } & \text { Central nervous system } \\ \text { CPP: } & \text { Conditioned place preference } \\ \text { ECM: } & \text { Extracellular matrix } \\ \text { METH: } & \text { Methamphetamine } \\ \text { MMP: } & \text { Matrix metalloproteinase } \\ \text { NAc: } & \text { Nucleus accumbens } \\ \text { NMDA: } & \text { N-methyl-D-aspartate } \\ \text { TIMP: } & \text { Tissue inhibitor of metalloproteinase } \\ \text { TrkB: } & \text { Tropomyosin-related kinase B. }\end{array}$

\section{Acknowledgments}

This study was supported in part by Grants-in-aid for Scientific Research (nos. 21790068, 22390046, 23790082, and 23659135) from the Japan Society for the Promotion of Science, a grant from the Japan Epilepsy Research Foundation, a grant from the Kowa Life Science Foundation, a grant from the Nakatomi Foundation, global COE program grants from the Ministry of Education, Culture, Sports, Science, and Technology of Japan and the Academic Frontier Project for Private Universities, and a matching fund subsidy from MEXT, 2007-2011.

\section{References}

[1] V. W. Yong, C. Power, P. Forsyth, and D. R. Edwards, "Metalloproteinases in biology and pathology of the nervous system," Nature Reviews Neuroscience, vol. 2, no. 7, pp. 502-511, 2001.

[2] I. M. Ethell and D. W. Ethell, "Matrix metalloproteinases in brain development and remodeling: synaptic functions and targets," Journal of Neuroscience Research, vol. 85, no. 13, pp. 2813-2823, 2007.

[3] M. D. Sternlicht and Z. Werb, "How matrix metalloproteinases regulate cell behavior," Annual Review of Cell and Developmental Biology, vol. 17, pp. 463-516, 2001.

[4] F. Mannello and G. Gazzanelli, "Tissue inhibitors of metalloproteinases and programmed cell death: conundrums, controversies and potential implications," Apoptosis, vol. 6, no. 6, pp. 479-482, 2001.

[5] C. Vaillant, M. Didier-Bazès, A. Hutter, M. F. Belin, and N. Thomasset, "Spatiotemporal expression patterns of metalloproteinases and their inhibitors in the postnatal developing rat cerebellum," Journal of Neuroscience, vol. 19, no. 12, pp. 49945004, 1999.

[6] J. Dzwonek, M. Rylski, and L. Kaczmarek, "Matrix metalloproteinases and their endogenous inhibitors in neuronal 
physiology of the adult brain," FEBS Letters, vol. 567, no. 1, pp. 129-135, 2004.

[7] J. W. Wright, J. R. Reichert, C. J. Davis, and J. W. Harding, "Neural plasticity and the brain renin-angiotensin system," Neuroscience and Biobehavioral Reviews, vol. 26, no. 5, pp. 529-552, 2002.

[8] S. Rivera, E. Tremblay, S. Timsit, O. Canals, Y. Ben-Ari, and M. Khrestchatisky, "Tissue inhibitor of metalloproteinases-1 (TIMP-1) is differentially induced in neurons and astrocytes after seizures: evidence for developmental, immediate early gene, and lesion response," Journal of Neuroscience, vol. 17, no. 11, pp. 4223-4235, 1997.

[9] E. Nedivi, D. Hevroni, D. Naot, D. Israell, and Y. Citri, "Numerous candidate plasticity-related genes revealed by differential cDNA cloning," Nature, vol. 363, no. 6431, pp. 718$722,1993$.

[10] A. Szklarczyk, J. Lapinska, M. Rylski, R. D. G. McKay, and L. Kaczmarek, "Matrix metalloproteinase-9 undergoes expression and activation during dendritic remodeling in adult hippocampus," Journal of Neuroscience, vol. 22, no. 3, pp. 920 930, 2002.

[11] V. Nagy, O. Bozdagi, A. Matynia et al., "Matrix metalloproteinase-9 is required for hippocampal late-phase long-term potentiation and memory," Journal of Neuroscience, vol. 26, no. 7, pp. 1923-1934, 2006.

[12] P. Okulski, T. M. Jay, J. Jaworski et al., "TIMP-1 abolishes MMP-9-dependent long-lasting long-term potentiation in the prefrontal cortex," Biological Psychiatry, vol. 62, no. 4, pp. 359 362, 2007.

[13] T. E. Brown, A. R. Wilson, D. L. Cocking, and B. A. Sorg, "Inhibition of matrix metalloproteinase activity disrupts reconsolidation but not consolidation of a fear memory," Neurobiology of Learning and Memory, vol. 91, no. 1, pp. 66-72, 2009.

[14] J. W. Wright, T. E. Brown, and J. W. Harding, "Inhibition of hippocampal matrix metalloproteinase-3 and -9 disrupts spatial memory," Neural Plasticity, vol. 2007, Article ID 73813, 2007.

[15] S. E. Meighan, P. C. Meighan, P. Choudhury et al., "Effects of extracellular matrix-degrading proteases matrix metalloproteinases 3 and 9 on spatial learning and synaptic plasticity," Journal of Neurochemistry, vol. 96, no. 5, pp. 1227-1241, 2006.

[16] F. A. Chaillan, S. Rivera, E. Marchetti et al., "Involvement of tissue inhibition of metalloproteinases-1 in learning and memory in mice," Behavioural Brain Research, vol. 173, no. 2, pp. 191-198, 2006.

[17] R. Pawlak, A. M. Magarinos, J. Melchor, B. McEwen, and S. Strickland, "Tissue plasminogen activator in the amygdala is critical for stress-induced anxiety-like behavior," Nature Neuroscience, vol. 6, no. 2, pp. 168-174, 2003.

[18] T. Matys, R. Pawlak, E. Matys, C. Pavlides, B. S. McEwen, and S. Strickland, "Tissue plasminogen activator promotes the effects of corticotropin- releasing factor on the amygdala and anxiety-like behavior," Proceedings of the National Academy of Sciences of the United States of America, vol. 101, no. 46, pp. 16345-16350, 2004.

[19] P. Michaluk, L. Mikasova, L. Groc, R. Frischknecht, D. Choquet, and L. Kaczmarek, "Matrix metalloproteinase-9 controls NMDA receptor surface diffusion through integrin $\beta 1$ signaling," Journal of Neuroscience, vol. 29, no. 18, pp. 6007-6012, 2009.

[20] C. Barkus, S. B. McHugh, R. Sprengel, P. H. Seeburg, J. N. P. Rawlins, and D. M. Bannerman, "Hippocampal NMDA receptors and anxiety: at the interface between cognition and emotion," European Journal of Pharmacology, vol. 626, no. 1, pp. 49-56, 2010.

[21] H. Mizoguchi, D. Ibi, K. Takuma et al., "Alterations of emotional and cognitive behaviors in matrix metalloproteinase-2 and -9-deficient mice," Open Behavioral Science Journal, vol. 4, pp. 19-25, 2010.

[22] D. Sulzer, "How addictive drugs disrupt presynaptic dopamine neurotransmission," Neuron, vol. 69, no. 4, pp. 628-649, 2011.

[23] M. S. Bowers, B. T. Chen, and A. Bonci, "AMPA receptor synaptic plasticity induced by psychostimulants: the past, present, and therapeutic future," Neuron, vol. 67, no. 1, pp. 11-24, 2010.

[24] K. M. Grant, T. D. LeVan, S. M. Wells et al., "Methamphetamine-associatedpsychosis," Journal of Neuroimmune Pharmacology. In press.

[25] H. Kamei, T. Nagai, H. Nakano et al., "Repeated methamphetamine treatment impairs recognition memory through a failure of novelty-induced ERK1/2 activation in the prefrontal cortex of mice," Biological Psychiatry, vol. 59, no. 1, pp. 75-84, 2006.

[26] H. Mizoguchi, K. Takuma, A. Fukakusa et al., "Improvement by minocycline of methamphetamine-induced impairment of recognition memory in mice," Psychopharmacology, vol. 196, no. 2, pp. 233-241, 2008.

[27] A. Nakajima, K. Yamada, T. Nagai et al., "Role of tumor necrosis factor- $\alpha$ in methamphetamine-induced drug dependence and neurotoxicity," Journal of Neuroscience, vol. 24, no. 9, pp. 2212-2225, 2004.

[28] H. Mizoguchi, K. Yamada, M. Mizuno et al., "Regulations of methamphetamine reward by extracellular signal-regulated kinase 1/2/ets-like gene-1 signaling pathway via the activation of dopamine receptors," Molecular Pharmacology, vol. 65, no. 5, pp. 1293-1301, 2004.

[29] T. E. Robinson and B. Kolb, "Persistent structural modifications in nucleus accumbens and prefrontal cortex neurons produced by previous experience with amphetamine," Journal of Neuroscience, vol. 17, no. 21, pp. 8491-8497, 1997.

[30] E. J. Nestler, "Molecular basis of long-term plasticity underlying addiction," Nature Reviews Neuroscience, vol. 2, no. 2, pp. 119-128, 2001.

[31] K. Yamada and T. Nabeshima, "Endogenous modulators for drug dependence," Biological and Pharmaceutical Bulletin, vol. 31, no. 9, pp. 1635-1638, 2008.

[32] H. Mizoguchi, K. Yamada, A. Mouri et al., "Role of matrix metalloproteinase and tissue inhibitor of MMP in methamphetamine-induced behavioral sensitization and reward: implications for dopamine receptor down-regulation and dopamine release," Journal of Neurochemistry, vol. 102, no. 5, pp. 1548-1560, 2007.

[33] H. Mizoguchi, K. Yamada, M. Niwa et al., "Reduction of methamphetamine-induced sensitization and reward in matrix metalloproteinase-2 and -9-deficient mice," Journal of Neurochemistry, vol. 100, no. 6, pp. 1579-1588, 2007.

[34] H. Mizoguchi, K. Yamada, and T. Nabeshima, "Neuropsychotoxicity of abused drugs: involvement of matrix metalloproteinase- 2 and -9 and tissue inhibitor of matrix metalloproteinase-2 in methamphetamine-induced behavioral sensitization and reward in rodents," Journal of Pharmacological Sciences, vol. 106, no. 1, pp. 9-14, 2008.

[35] C. Vaillant, M. Didier-Bazès, A. Hutter, M. F. Belin, and N. Thomasset, "Spatiotemporal expression patterns of metalloproteinases and their inhibitors in the postnatal developing rat cerebellum," Journal of Neuroscience, vol. 19, no. 12, pp. 4994-5004, 1999. 
[36] T. M. Reeves, M. L. Prins, J. Zhu, J. T. Povlishock, and L. L. Phillips, "Matrix metalloproteinase inhibition alters functional and structural correlates of deafferentation-induced sprouting in the dentate gyrus," Journal of Neuroscience, vol. 23, no. 32, pp. 10182-10189, 2003.

[37] T. E. Brown, M. R. Forquer, D. L. Cocking, H. T. Jansen, J. W. Harding, and B. A. Sorg, "Role of matrix metalloproteinases in the acquisition and reconsolidation of cocaine-induced conditioned place preference," Learning and Memory, vol. 14, no. 3, pp. 214-223, 2007.

[38] T. E. Brown, M. R. Forquer, J. W. Harding, J. W. Wright, and B. A. Sorg, "Increase in matrix metalloproteinase-9 levels in the rat medial prefrontal cortex after cocaine reinstatement of conditioned place preference," Synapse, vol. 62, no. 12, pp. 886-889, 2008.

[39] P. Sillanaukee, A. Kalela, K. Seppä, M. Höyhtyä, and S. T. Nikkari, "Matrix metalloproteinase-9 is elevated in serum of alcohol abusers," European Journal of Clinical Investigation, vol. 32, no. 4, pp. 225-229, 2002.

[40] J. Haorah, K. Schall, S. H. Ramirez, and Y. Persidsky, "Activation of protein tyrosine kinases and matrix metalloproteinases causes blood-brain barrier injury: novel mechanism for neurodegeneration associated with alcohol abuse," GLIA, vol. 56, no. 1, pp. $78-88,2008$.

[41] A. W. Smith, K. A. Nealey, J. W. Wright, and B. M. Walker, "Plasticity associated with escalated operant ethanol selfadministration during acute withdrawal in ethanol-dependent rats requires intact matrix metalloproteinase systems," Neurobiology of Learning and Memory, vol. 96, no. 2, pp. 199-206, 2011.

[42] Y. Liu, S. Brown, J. Shaikh, J. A. Fishback, and R. R. Matsumoto, "Relationship between methamphetamine exposure and matrix metalloproteinase 9 expression," NeuroReport, vol. 19, no. 14, pp. 1407-1409, 2008.

[43] K. Conant, I. Lonskaya, A. Szklarczyk et al., "Methamphetamine-associated cleavage of the synaptic adhesion molecule intercellular adhesion molecule-5," Journal of Neurochemistry, vol. 118, no. 4, pp. 521-532, 2011.

[44] D. Sulzer, T. K. Chen, Y. Y. Lau, H. Kristensen, S. Rayport, and A. Ewing, "Amphetamine redistributes dopamine from synaptic vesicles to the cytosol and promotes reverse transport," Journal of Neuroscience, vol. 15, no. 5, pp. 4102-4108, 1995.

[45] T. Nagai, Y. Noda, K. Ishikawa et al., "The role of tissue plasminogen activator in methamphetamine-related reward and sensitization," Journal of Neurochemistry, vol. 92, no. 3, pp. 660-667, 2005.

[46] H. Khoshbouei, N. Sen, B. Guptaroy et al., "N-terminal phosphorylation of the dopamine transporter is required for amphetamine-induced efflux," PLoS Biology, vol. 2, no. 3, pp. 387-393, 2004.

[47] R. Z. Goldstein and N. D. Volkow, "Drug addiction and its underlying neurobiological basis: neuroimaging evidence for the involvement of the frontal cortex," American Journal of Psychiatry, vol. 159, no. 10, pp. 1642-1652, 2002.

[48] M. S. Bowers, K. McFarland, R. W. Lake et al., "Activator of G protein signaling 3: a gatekeeper of cocaine sensitization and drug seeking," Neuron, vol. 42, no. 2, pp. 269-281, 2004.

[49] J. F. Bowyer and N. Weiner, "Modulation of the $\mathrm{Ca}^{++}$ evoked release of $[3 \mathrm{H}]$ dopamine from striatal synaptosomes by dopamine (D2) agonists and antagonists," Journal of Pharmacology and Experimental Therapeutics, vol. 241, no. 1, pp. 27-33, 1987.
[50] N. Lindgren, Z. Q. D. Xu, M. Herrera-Marschitz, J. Haycock, T. Hökfelt, and G. Fisone, "Dopamine D2 receptors regulate tyrosine hydroxylase activity and phosphorylation at Ser40 in rat striatum," European Journal of Neuroscience, vol. 13, no. 4, pp. 773-780, 2001.

[51] A. M. Palmer, "Neuroprotective therapeutics for Alzheimers disease: progress and prospects," Trends in Pharmacological Sciences, vol. 32, no. 3, pp. 141-147, 2011.

[52] K. Yamada and T. Nabeshima, "Animal models of Alzheimer's disease and evaluation of anti-dementia drugs," Pharmacology and Therapeutics, vol. 88, no. 2, pp. 93-113, 2000.

[53] K. Takuma, S. S. Yan, D. M. Stern, and K. Yamada, "Mitochondrial dysfunction, endoplasmic reticulum stress, and apoptosis in Alzheimer's disease," Journal of Pharmacological Sciences, vol. 97, no. 3, pp. 312-316, 2005.

[54] K. Takuma, F. Fang, W. Zhang et al., "RAGE-mediated signaling contributes to intraneuronal transport of amyloid- $\beta$ and neuronal dysfunction," Proceedings of the National Academy of Sciences of the United States of America, vol. 106, no. 47, pp. 20021-20026, 2009.

[55] K. Yamada, M. Takayanagi, H. Kamei et al., "Effects of memantine and donepezil on amyloid $\beta$-induced memory impairment in a delayed-matching to position task in rats," Behavioural Brain Research, vol. 162, no. 2, pp. 191-199, 2005.

[56] D. Wang, Y. Noda, Y. Zhou et al., "The allosteric potentiation of nicotinic acetylcholine receptors by galantamine ameliorates the cognitive dysfunction in beta amyloid 25-35 I.c.v.-injected mice: involvement of dopaminergic systems," Neuropsychopharmacology, vol. 32, no. 6, pp. 1261-1271, 2007.

[57] T. Alkam, A. Nitta, H. Mizoguchi, A. Itoh, and T. Nabeshima, "A natural scavenger of peroxynitrites, rosmarinic acid, protects against impairment of memory induced by A $\beta 25-35$," Behavioural Brain Research, vol. 180, no. 2, pp. 139-145, 2007.

[58] M. H. Tran, K. Yamada, A. Olariu, M. Mizuno, X. H. Ren, and T. Nabeshima, "Amyloid beta-peptide induces nitric oxide production in rat hippocampus: association with cholinergic dysfunction and amelioration by inducible nitric oxide synthase inhibitors," The FASEB Journal, vol. 15, no. 8, pp. 14071409, 2001.

[59] K. Yamada, T. Tanaka, D. Han, K. Senzaki, T. Kameyama, and T. Nabeshima, "Protectiye effects of idebenone and $\alpha$-tocopherol on $\beta$-amyloid-(1-42)-induced learning and memory deficits in rats: implication of oxidative stress in $\beta$-amyloidinduced neurotoxicity in vivo," European Journal of Neuroscience, vol. 11, no. 1, pp. 83-90, 1999.

[60] C. Zussy, A. Brureau, B. Delair et al., "Time-course and regional analyses of the physiopathological changes induced after cerebral injection of an amyloid $\beta$ fragment in rats," American Journal of Pathology, vol. 179, no. 1, pp. 315-334, 2011.

[61] T. Malm, M. Ort, L. Tähtivaara et al., " $\beta$-amyloid infusion results in delayed and age-dependent learning deficits without role of inflammation or $\beta$-amyloid deposits," Proceedings of the National Academy of Sciences of the United States of America, vol. 103, no. 23, pp. 8852-8857, 2006.

[62] J. R. Backstrom, G. P. Lim, M. J. Cullen, and Z. A. Tökés, "Matrix metalloproteinase-9 (MMP-9) is synthesized in neurons of the human hippocampus and is capable of degrading the amyloid- $\beta$ peptide (1-40)," Journal of Neuroscience, vol. 16, no. 24, pp. 7910-7919, 1996.

[63] J. R. Backstrom, C. A. Miller, and Z. A. Tokes, "Characterization of neutral proteinases from Alzheimer-affected and control brain specimens: identification of calcium-dependent metalloproteinases from the hippocampus," Journal of Neurochemistry, vol. 58, no. 3, pp. 983-992, 1992. 
[64] S. Baig, P. G. Kehoe, and S. Love, "MMP-2, -3 and -9 levels and activity are not related to $\mathrm{A} \beta$ load in the frontal cortex in Alzheimer's disease," Neuropathology and Applied Neurobiology, vol. 34, no. 2, pp. 205-215, 2008.

[65] M. Asahina, Y. Yoshiyama, and T. Hattori, "Expression of matrix metalloproteinase- 9 and urinary-type plasminogen activator in Alzheimer's disease brain," Clinical Neuropathology, vol. 20, no. 2, pp. 60-63, 2001.

[66] S. Deb and P. E. Gottschall, "Increased production of matrix metalloproteinases in enriched astrocyte and mixed hippocampal cultures treated with $\beta$-amyloid peptides," Journal of Neurochemistry, vol. 66, no. 4, pp. 1641-1647, 1996.

[67] P. Yan, X. Hu, H. Song et al., "Matrix metalloproteinase-9 degrades amyloid- $\beta$ fibrils in vitro and compact plaques in situ," Journal of Biological Chemistry, vol. 281, no. 34, pp. 2456624574, 2006.

[68] S. Deb, J. W. Zhang, and P. E. Gottschall, " $\beta$-amyloid induces the production of active, matrix-degrading proteases in cultured rat astrocytes," Brain Research, vol. 970, no. 1-2, pp. 205-213, 2003.

[69] E. M. Muir, K. H. Adcock, D. A. Morgenstern et al., "Matrix metalloproteases and their inhibitors are produced by overlapping populations of activated astrocytes," Molecular Brain Research, vol. 100, no. 1-2, pp. 103-117, 2002.

[70] H. Mizoguchi, K. Takuma, E. Fukuzaki et al., "Matrix metalloprotease-9 inhibition improves amyloid $\beta$-mediated cognitive impairment and neurotoxicity in mice," Journal of Pharsmacology and Experimental Therapeutics, vol. 331, no. 1, pp. 14-22, 2009.

[71] J. Jourquin, E. Tremblay, N. Décanis et al., "Neuronal activitydependent increase of net matrix metalloproteinase activity is associated with MMP-9 neurotoxicity after kainate," European Journal of Neuroscience, vol. 18, no. 6, pp. 1507-1517, 2003.

[72] Y. Tamura, F. Watanabe, T. Nakatani et al., "Highly selective and orally active inhibitors of type IV collagenase (MMP-9 and MMP-2): N-sulfonylamino acid derivatives," Journal of Medicinal Chemistry, vol. 41, no. 4, pp. 640-649, 1998.

[73] L. H. Zeng, L. Xu, N. R. Rensing, P. M. Sinatra, S. M. Rothman, and M. Wong, "Kainate seizures cause acute dendritic injury and actin depolymerization in vivo," Journal of Neuroscience, vol. 27, no. 43, pp. 11604-11613, 2007.

[74] M. Kokaia, "Seizure-induced neurogenesis in the adult brain," European Journal of Neuroscience, vol. 33, no. 6, pp. 1133$1138,2011$.

[75] D. Han, K. Yamada, K. Senzaki, H. Xiong, H. Nawa, and T. Nabeshima, "Involvement of nitric oxide in pentylenetetrazole-induced kindling in rats," Journal of Neurochemistry, vol. 74, no. 2, pp. 792-798, 2000.

[76] A. Wahab, K. Albus, S. Gabriel, and U. Heinemann, "In search of models of pharmacoresistant epilepsy," Epilepsia, vol. 51, no. 3, pp. 154-159, 2010.

[77] W. Löscher and R. Köhling, "Functional, metabolic, and synaptic changes after seizures as potential targets for antiepileptic therapy," Epilepsy and Behavior, vol. 19, no. 2, pp. 105-113, 2010.

[78] N. Suenaga, T. Ichiyama, M. Kubota, H. Isumi, J. Tohyama, and S. Furukawa, "Roles of matrix metalloproteinase- 9 and tissue inhibitors of metalloproteinases 1 in acute encephalopathy following prolonged febrile seizures," Journal of the Neurological Sciences, vol. 266, no. 1-2, pp. 126-130, 2008.

[79] M. Rylski, R. Amborska, K. Zybura et al., "JunB is a repressor of MMP-9 transcription in depolarized rat brain neurons," Molecular and Cellular Neuroscience, vol. 40, no. 1, pp. 98-110, 2009.
[80] F. A. Konopacki, M. Rylski, E. Wilczek et al., "Synaptic localization of seizure-induced matrix metalloproteinase- 9 mRNA," Neuroscience, vol. 150, no. 1, pp. 31-39, 2007.

[81] J. W. Wright and J. W. Harding, "The brain angiotensin system and extracellular matrix molecules in neural plasticity, learning, and memory," Progress in Neurobiology, vol. 72, no. 4, pp. 263-293, 2004.

[82] J. W. Zhang, S. Deb, and P. E. Gottschall, "Regional and differential expression of gelatinases in rat brain after systemic kainic acid or bicuculline administration," European Journal of Neuroscience, vol. 10, no. 11, pp. 3358-3368, 1998.

[83] G. M. Wilczynski, F. A. Konopacki, E. Wilczek et al., "Important role of matrix metalloproteinase 9 in epileptogenesis," Journal of Cell Biology, vol. 180, no. 5, pp. 1021-1035, 2008.

[84] G. W. Kim, H. J. Kim, K. J. Cho, H. W. Kim, Y. J. Cho, and B. I. Lee, "The role of MMP-9 in integrin-mediated hippocampal cell death after pilocarpine-induced status epilepticus," Neurobiology of Disease, vol. 36, no. 1, pp. 169-180, 2009.

[85] E. Takács, R. Nyilas, Z. Szepesi et al., "Matrix metalloproteinase- 9 activity increased by two different types of epileptic seizures that do not induce neuronal death: a possible role in homeostatic synaptic plasticity," Neurochemistry International, vol. 56, no. 6-7, pp. 799-809, 2010.

[86] H. Mizoguchi, J. Nakade, M. Tachibana et al., "Matrix metalloproteinase- 9 contributes to kindled seizure development in pentylenetetrazole-treated mice by converting pro-BDNF to mature BDNF in the hippocampus," Journal of Neuroscience, vol. 31, no. 36, pp. 12963-12971, 2011.

[87] R. J. Cabelli, A. Hohn, and C. J. Shatz, "Inhibition of ocular dominance column formation by infusion of NT-4/5 or BDNF," Science, vol. 267, no. 5204, pp. 1662-1666, 1995.

[88] H. W. Horch, A. Krüttgen, S. D. Portbury, and L. C. Katz, "Destabilization of cortical dendrites and spines by BDNF," Neuron, vol. 23, no. 2, pp. 353-364, 1999.

[89] G. Nagappan, E. Zaitsev, V. V. Senatorov Jr., J. Yang, B. L. Hempstead, and B. Lu, "Control of extracellular cleavage of ProBDNF by high frequency neuronal activity," Proceedings of the National Academy of Sciences of the United States of America, vol. 106, no. 4, pp. 1267-1272, 2009.

[90] R. Lee, P. Kermani, K. K. Teng, and B. L. Hempstead, "Regulation of cell survival by secreted proneurotrophins," Science, vol. 294, no. 5548, pp. 1945-1948, 2001.

[91] J. H. Jung, M. H. Park, S. Y. Choi, and J. Y. Koh, "Activation of the Trk signaling pathway by extracellular zinc. Role of metalloproteinases," Journal of Biological Chemistry, vol. 280, no. 12, pp. 11995-12001, 2005.

[92] R. Koyama, M. K. Yamada, S. Fujisawa, R. Katoh-Semba, N. Matsuki, and Y. Ikegaya, "Brain-derived neurotrophic factor induces hyperexcitable reentrant circuits in the dentate gyrus," Journal of Neuroscience, vol. 24, no. 33, pp. 7215-7224, 2004. 

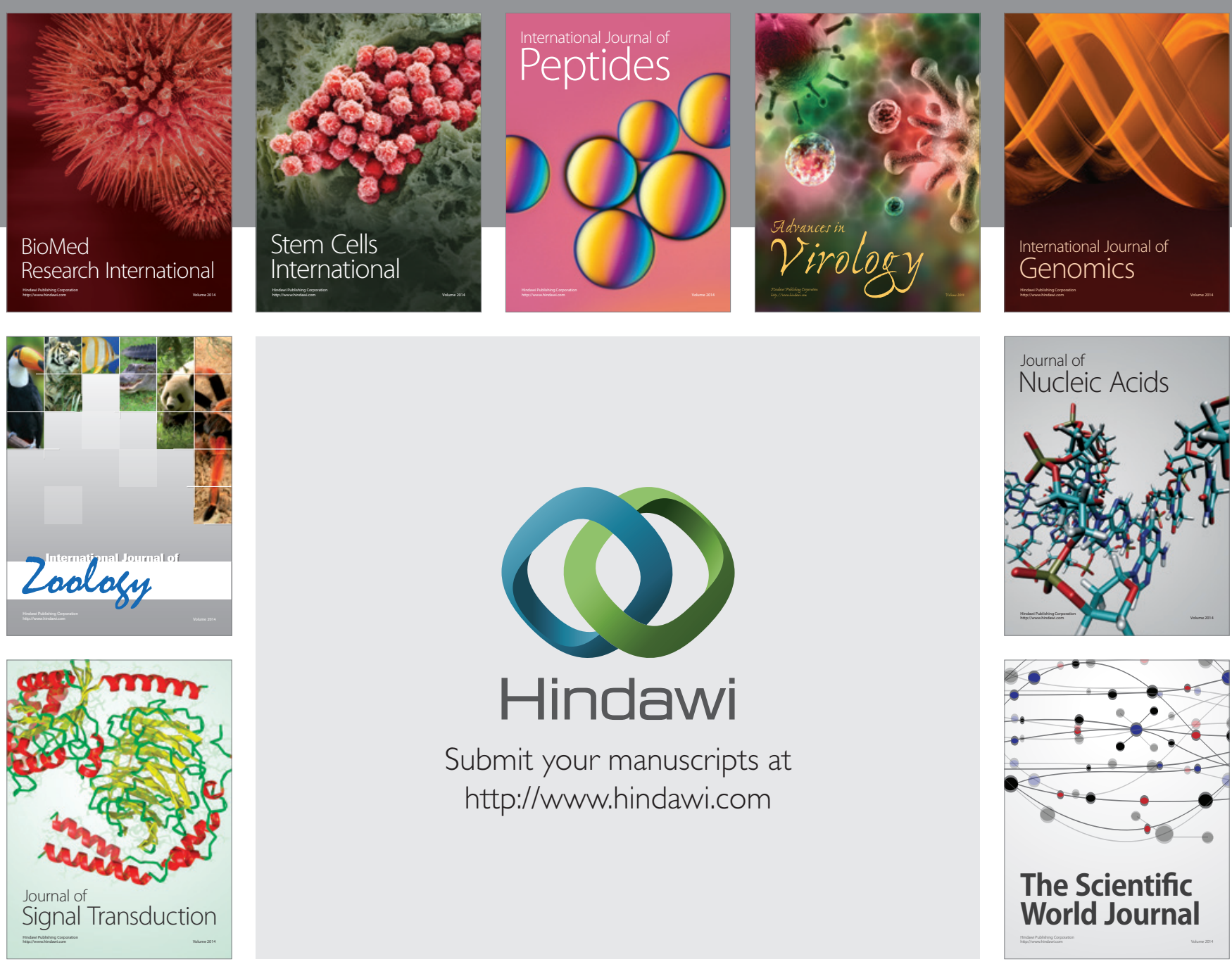

Submit your manuscripts at

http://www.hindawi.com
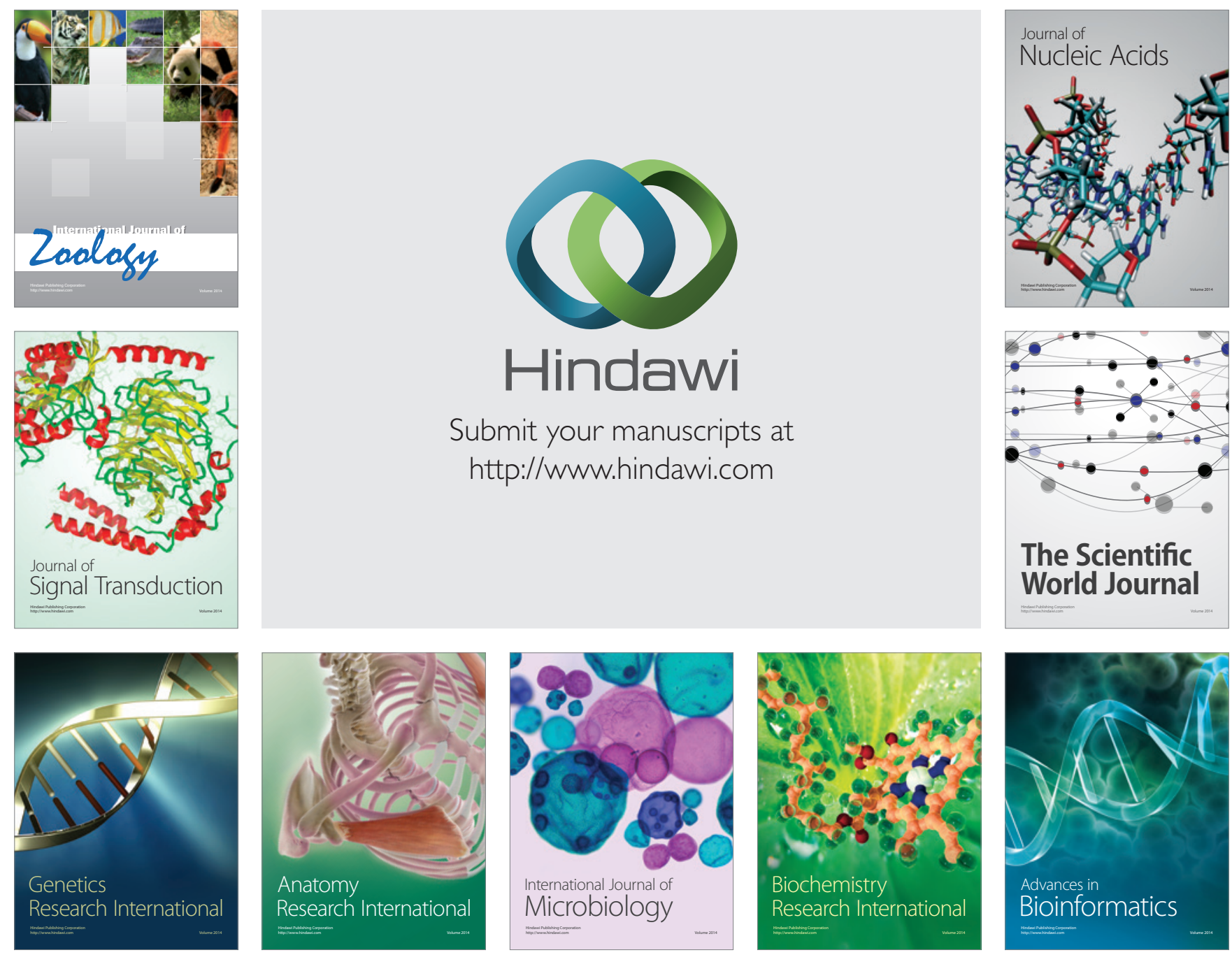

The Scientific World Journal
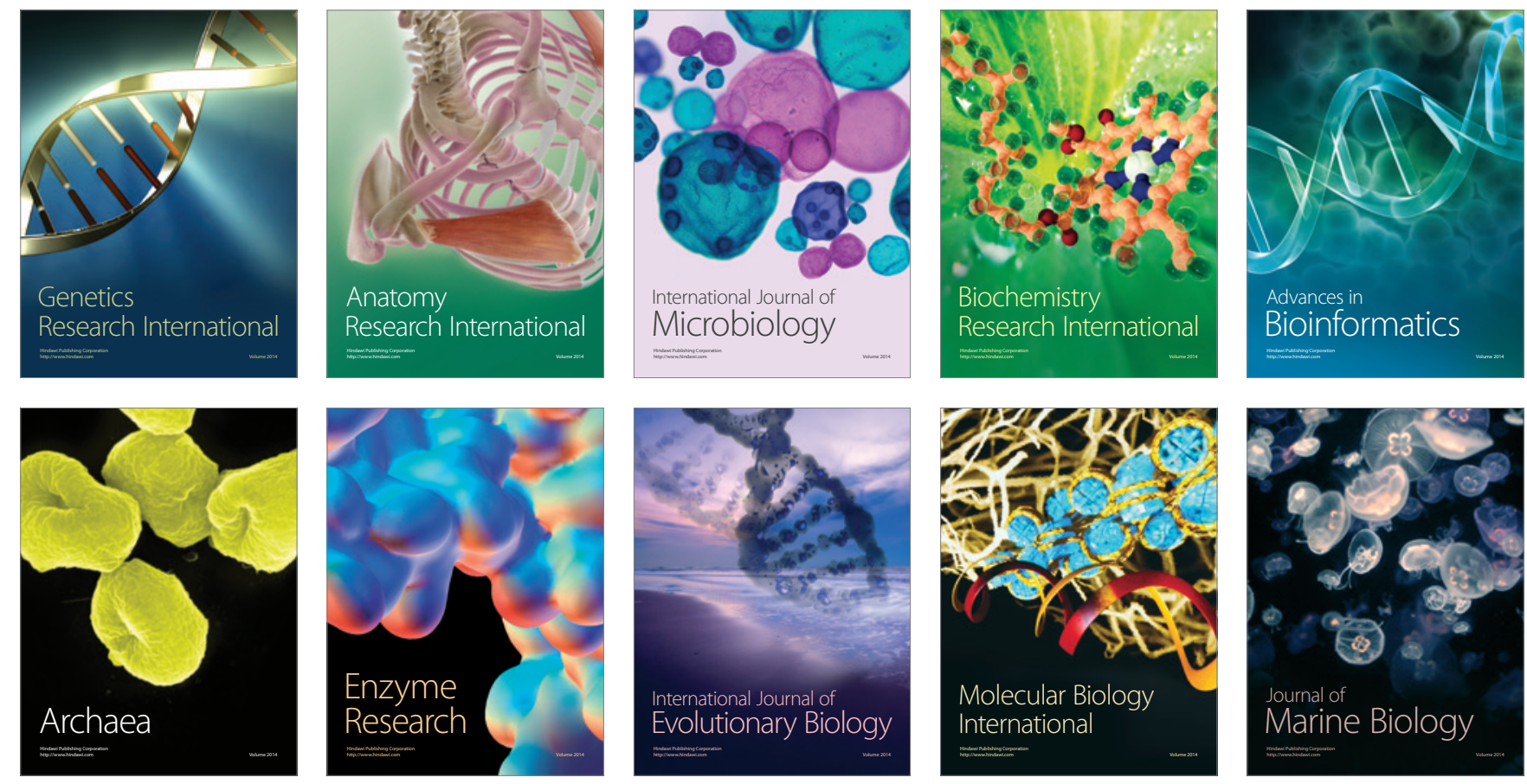